\title{
EL MOBBING Y LA TEORÍA DE LA ACCIÓN DE PIERRE BOURDIEU
}

\section{MOBBING AND THE ACTION THEORY OF PIERRE BOURDIEU}

\author{
Susana Mayoral Blasco \\ Universidad de Zaragoza. España \\ smayoral@unizar.es
}

\begin{abstract}
RESUMEN
En la primera parte del articulo fundamentamos teóricamente por qué abordar el estudio del mobbing desde la perspectiva de la Teoría de la Acción de Pierre Bourdieu y realizamos una revisión de los principales conceptos de su teoría: Campo, Capital y Habitus. En la segunda parte presentamos el modelo y ponemos estos conceptos en relación con los factores asociados al mobbing, obteniendo una comprensión más profunda y compleja del fenómeno que la que ofrecen modelos alternativos como el psicopatológico de lñaki Piñuel, el psicosocial de Jorge Corsi y Graciela Peyrý y el de prevención de riesgos laborales de Dolores Fernández y Clara Llorens.
\end{abstract}

\section{Palabras Clave}

Campo, Capital, Habitus, Práctica.

\begin{abstract}
In the first part of this article we defend theoretically why to undertake the study of mobbing from the perspective of Pierre Bourdieu's Action Theory Model. At the same time we revise the main concepts of his theory, that is, Field, Capital and Habitus In the second part we present the model linking these concepts with the factors that are associated with mobbing in order to get a deeper and more complex understanding of the phenomenon than those of the psychopathological model developed by Iñaki Piñuel, the socio-psychological model by Jorge Corsi and Graciela Peyrú, and the job hazard model by Dolores Fernandez and Clara Llorens.
\end{abstract}

\section{KEYWORDS}

Capital, Field, Habitus, Practice. 


\section{INTRODUCCIÓN}

En este artículo proponemos abordar el estudio del mobbing a través del modelo de análisis proporcionado por la Teoría de la Acción Práctica de Pierre Bourdieu (2002) donde:

$$
\text { PRÁCTICA }=\text { CAMPO + [(CAPITAL) }(\text { Habitus })]
$$

Para ello, fundamentamos teóricamente nuestra propuesta y realizamos una revisión de los conceptos de Campo, Capital y Habitus, poniendo estos conceptos en relación con los factores asociados al mobbing. Tras haber contrastado empíricamente la potencia explicativa del modelo de Bourdieu respecto a los otros modelos, proponemos el uso de este modelo para explicar el fenómeno.

Lo hacemos con los siguientes objetivos: primero, introducir la perspectiva sociológica en el análisis del mobbing; segundo, proponer un modelo que dé cuenta del mobbing en mayor medida de lo que lo hacen los modelos teóricos que habitualmente se utilizan para su análisis en el ámbito sindical español. Estos modelos, corresponden a la perspectiva psicológica: Modelo Psicopatológico de Iñaki Piñuel (2001); Modelo Psicosocial de Jorge Corsi y Graciela Peyrú (2003); y la perspectiva de la Prevención de los Riesgos Laborales (Modelo Factores de Riesgo Psicosocial + Factores de Acoso = Daños a la Salud), de Dolores Fernández y Clara Llorens (2002).

Nuestro planteamiento es que estos modelos son útiles, puesto que contribuyen a explicar el fenómeno, pero parciales, ya que consideramos que contribuyen a explicar determinadas facetas del fenómeno, pero no éste en toda su complejidad. Consideramos que una de las razones de ello, es que estos modelos explican el mobbing privilegiando determinados factores, como son el perfil personal (Piñuel 2001), las dinámicas de violencia grupal (Corsi y Peyrú 2003) o la existencia de riesgos psicosociales (Fernández y Llorens 2002). Consideramos además que estos modelos no incorporan de forma suficientemente satisfactoria factores que parecen ser importantes en la existencia del mobbing, como el Poder o la Cultura de la Empresa.

Como metodología para contrastar la mayor potencia explicativa del modelo de Bourdieu y de los conceptos de Campo, Habitus y Capital, hemos usado la metodología cualitativa, y como técnica de investigación, el estudio de caso.

Como principales resultados mostramos en este artículo la mayor potencia explicativa del modelo de Bourdieu, así como que los conceptos de Campo, Capital y Habitus son lo suficientemente flexibles y amplios para incorporar factores relacionados con la existencia del mobbing que ya habían sido detectados por los autores mencionados (Piñuel 2001; Corsi y Peyrú 2002; Fernández y Llorens 2002), así como para incorporar factores nuevos, como son el Poder, el contexto, o la cultura de la empresa. 


\section{VALORACIÓN DE LOS MODELOS ANALÍTICOS}

A partir de investigaciones empíricas propias, pudimos detectar que los modelos explicativos del mobbing, que se estaban utilizando desde la acción sindical en España, no daban cuenta del fenómeno en toda su complejidad. Nuestra valoración es que todos estos modelos son útiles, puesto que contribuyen a explicar el fenómeno, pero al mismo tiempo son parciales, ya que explican facetas del fenómeno, pero no lo explican en toda su complejidad.

Cada uno de estos modelos explica el mobbing poniendo el acento en determinados factores, como son el perfil personal del acosadorla y su víctima - Modelo Psicopatológico de Piñuel (2001) - las dinámicas de violencia grupal —Modelo Psicosocial de Corsi y Peyrú (2003) - o la existencia de riesgos laborales -Modelo Factores de Riesgo Psicosocial + Factores de Acoso = Daños a la Salud, de Fernández y Llorens (2002)-, pero dejando en segundo plano factores que parecen ser claves, como el Poder y la Cultura de la Empresa.

Respecto al Poder, los autores y autoras que hemos mencionado reconocen la centralidad del factor Poder en la existencia del mobbing, e incluso incluyen la existencia de una relación asimétrica de Poder entre el acosador/a y víctima en sus definiciones de mobbing (Corsi y Peyrú 2003: 183; Fernández y Llorens 2002: 2). Sin embargo, una vez reconocida la importancia del Poder, los autores y autoras no incluyen este factor de forma fuerte en sus modelos teóricos, que parecen seguir la siguiente lógica en su construcción: "partiendo de que existe una relación asimétrica de Poder entre el acosador/a y la víctima, vamos a explicar por qué se produce el mobbing", dejando fuera de sus modelos este factor. Nuestro planteamiento es que si un factor parece ser clave en la existencia de un fenómeno, debe integrarse dentro de cualquier modelo explicativo del fenómeno.

Asimismo, si la relación asimétrica de Poder entre acosador/a y víctima es fundamental para que pueda darse el mobbing, es decir, si el hecho de que el acosador/a tenga más Poder es lo que va a permitir efectuar el mobbing sobre la víctima, podemos considerar que el Poder es un Capital para ejercer el mobbing.

Otro factor que parece ser importante en la aparición y desarrollo del mobbing es la Cultura de la Empresa, según señalan autores de referencia en el tema del mobbing, como Jesús Pérez Bilbao (1998; 2001; 2002) o Concepción Saéz Navarro (2001) e instituciones como la Agencia Europea para la Seguridad y la Salud en el Trabajo (2002). El Ministerio de Trabajo español, en su Nota Técnica de Prevención 476 (Martín Daza y otros 1998) señala como la gestión del conflicto por parte de los superiores es un elemento clave en la resolución o no de los casos, lo que evidentemente podemos relacionar con la cultura de la empresa (estilos de mando y resolución de conflictos). Sin embargo, consideramos que los modelos citados no incorporan satisfactoriamente este aspecto. El Modelo Psicopatológico de Piñuel (2001: 132) menciona el "comportamiento organizativo" caracterizado por "la competitividad, prácticas no éticas, inseguridad" como un factor situacional, es decir, como telón de fondo del mobbing, ya que en su modelo el 
factor explicativo central lo constituye el perfil personal, tanto del acosador/a como de la víctima. El Modelo de la Psicología Social de Corsi y Peyrú (2003) conecta la existencia del caso de mobbing individual con la existencia de una dinámica grupal de violencia social, existente en el lugar de trabajo donde se da el mobbing, por lo que podemos considerar que sí incorpora la cultura de empresa al análisis del mobbing, y para estos autores constituye el factor explicativo central. Fernández y Llorens no la incluyen en su modelo de análisis.

En este sentido, la Cultura de la Empresa puede considerarse un Habitus Colectivo con manifestaciones individuales. Por lo tanto, el concepto de Habitus nos permitiría incorporar la cultura de la empresa al análisis del mobbing.

Respecto al "contexto", el Modelo Psicopatológico de Piñuel (2001: 132) menciona las formas de organización del trabajo (burocracia, desorganización, caos) como factor situacional, es decir, el contexto que facilita la aparición y desarrollo del mobbing. El Modelo de Fernández y Llorens (2002) privilegia claramente el contexto como factor explicativo del mobbing al relacionarlo con la organización del trabajo, de hecho, al relacionar directamente el mobbing y la existencia de riesgos psicosociales, al extremo de plantear que "el elemento fundamental es la organización del trabajo, puesto que si hay intención de causar daño pero la organización del trabajo no puede ser usada como elemento hostigador, no se dará el acoso moral" (Fernández y Llorens 2002: 3).

El concepto de "Campo" nos permite incorporar la importancia del contexto al análisis del mobbing.

\section{Modelo inicial propuesto}

Todo lo anterior nos lleva a plantear la necesidad de un modelo explicativo del mobbing, que incorpore estos factores: el Poder, el Contexto y la Cultura, no como telón de fondo, sino como factores explicativos de peso. Proponemos como modelo provisional capaz de integrar dentro estos factores, así como otros aportados por los modelos revisados, el Modelo de la Teoría de la Acción de Pierre Bourdieu (2002), donde:

$$
\text { PRÁCTICA }=[\text { CAMPO }+(\text { CAPITAL })(\text { Habitus })]
$$

Dado que este modelo ha demostrado su potencia para explicar otras realidades sociales, sugerimos que también es aplicable al análisis del mobbing. Por tanto, la originalidad de nuestra propuesta no reside tanto en elaborar un modelo nuevo, sino en abordar el análisis del mobbing desde una perspectiva sociológica basada en esta teoría. A continuación, presentamos gráficamente una primera adaptación de la formulación de Bourdieu (2002) al estudio del mobbing, a modo de hipótesis de partida. 


\section{Gráfico 1.}

Aplicación del modelo de la Teoría de la Acción de Bourdieu al análisis del mobbing.

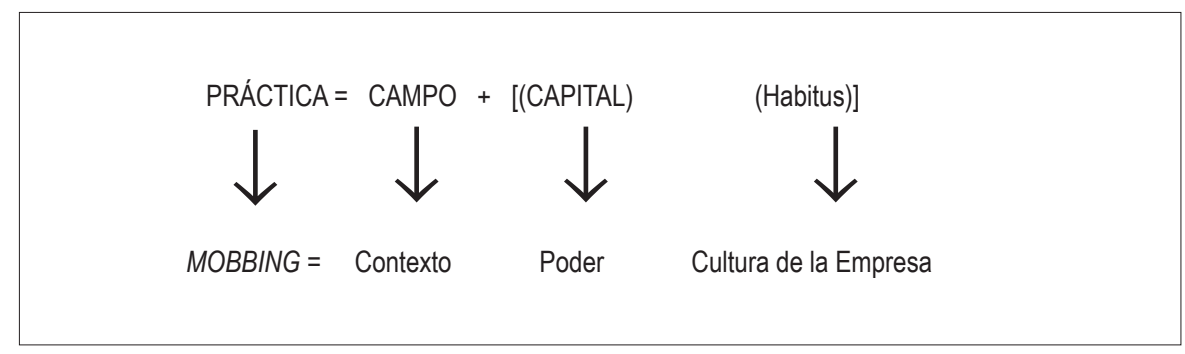

\section{¿Por qué abordar el estudio del mobbing desde la Teoría de la Acción Práctica DE BOURDIEU?}

Bourdieu nos va a aportar conceptos y perspectivas clave para abordar el estudio del mobbing. Su principal aportación será la misma que realiza al estudio de los diversos temas que trató a lo largo de su vida, es decir, su labor de síntesis y su perspectiva. En palabras del propio autor.

"Lo esencial de mi labor, lo más elemental y fundamental. Para empezar una Filosofía de la Ciencia que cabe llamar relacional en tanto que otorga la primacía a las relaciones. Aunque en opinión de autores tan diferentes como Cassier o Bachelard, esta filosofía sea la de toda la ciencia moderna, sólo se aplica en contadas ocasiones a las ciencias sociales, sin duda porque se opone muy directamente a las rutinas del pensamiento habitual (o semicientífico) del mundo social que se ocupa más de 'realidades' sustanciales, individuos, grupos, etc. que de relaciones objetivas que no se pueden mostrar ni tocar con la mano y que hay que conquistar, elaborar y validar a través de la labor científica. A continuación, una Filosofía de la Acción, designada a veces como disposicional, que toma en consideración las potencialidades inscritas en el cuerpo de los agentes y en la estructura de las situaciones en las que estas actúan, o con mayor exactitud en su relación.

Esta filosofía se condensa en un reducido número de conceptos fundamentales, Habitus, Campo y Capital, cuya piedra angular es la relación de doble sentido entre las estructuras objetivas (las de los campos sociales) y las estructuras incorporadas (los Habitus)".

(Bourdieu 2002: 7).

Respecto a lo que Bourdieu dio en llamar su Filosofia de la Ciencia, en la que lo real es relacional, lo real no se identifica con sustancias sino con relaciones. El mismo autor dice: 
"Los objetos ordinarios de la investigación son realidades designadas al investigador por el hecho de que 'se hacen notar'. De alguna manera 'crean problema', por ejemplo 'las madres solteras del ghetto negro de Chicago'. Y casi siempre los investigadores toman como objetos problemas que crean grupos de individuos más o menos arbitrariamente delimitados, obtenidos por particiones sucesivas de una categoría ella misma preconstruida, como 'los viejos', 'los jóvenes', 'los inmigrantes'”.

(Bourdieu 1997: 199).

Estableciendo un paralelismo, en nuestro caso las realidades que "se hacen notar" son el acosador/a, el acosado/a y el acoso. Sin embargo, Bourdieu nos advierte del peligro que esto representa: el riesgo de atribuir a los agentes que se hacen notar, que son visibles, unas propiedades (o características) que en realidad no son intrínsecas, sino que son el producto de la posición que ocupan en una red de relaciones. En su obra Cosas Dichas (Bourdieu 1996), el autor pone un ejemplo al referirse al estudio de las prácticas deportivas:

"En suma, la prioridad de las prioridades es la construcción de la estructura del espacio de las prácticas deportivas cuyos efectos registrarán las monografías consagradas a deportes particulares. Si no sé que las perturbaciones de Urano son determinadas por Neptuno creeré captar lo que pasa en Urano mientras que en realidad captaré lo que pasa en Neptuno".

(Bourdieu, 1996: 175).

El centrar nuestro estudio en el acosador/a y su víctima —-como hacen algunos autores desde la perspectiva psicopatológica, como Piñuel (2001)_, nos puede llevar a buscar y encontrar en el fragmento estudiado mecanismos o principios que en realidad están en el exterior del objeto analizado, en las relaciones con otros objetos. Nos pueden llevar por ejemplo a atribuir el mobbing "solamente" a los perfiles psicológicos del acosador y de su víctima.

De esta perspectiva teórica en la que lo real es relacional, se deriva una perspectiva metodológica:

"Hay allí un principio de método muy general: más que contentarse con conocer a fondo un pequeño sector de la realidad de la que no se sabe demasiado, por no haberse planteado la pregunta cómo se sitúa en el espacio de donde fue separado y lo que su funcionamiento debe a esta posición [...] es necesario, pues, a la manera de los arquitectos académicos que presentan un bosquejo a la carbonilla del conjunto del edificio en el interior del cual se situaba la parte elaborada en detalle, esforzarse en construir una descripción sumaria del conjunto del espacio considerado.

Este marco provisorio, por imperfecto que sea, se sabe por lo menos que debe llenarse y que los trabajos empíricos mismos que orienta contribuirán a llenarlo. Resulta que 
estos trabajos son radicalmente diferentes, en su misma intención, de los que hubieran sido en ausencia de ese marco que es la condición de una construcción adecuada de los objetos de la investigación empírica y concreta".

(Bourdieu 1996: 177).

Aplicado a nuestro caso, nos llevaría a plantearnos que quizá la posibilidad de ser una acosador/a o una víctima de mobbing, no esté en función de los perfiles psicológicos (o no sólo), sino que la posibilidad de ejercer como acosador/a o ser una víctima potencial dependen de la posición que se ocupe en una red de relaciones. En todo caso, nos plantea la necesidad de que para entender el mobbing es necesario situar éste dentro de una arquitectura más amplia, y no centrarnos sólo en el estudio de las realidades que se hacen notar. Como lo real es relacional, es necesario pensar relacionalmente lo real como perspectiva metodológica básica.

Para Bourdieu es necesario escapar tanto de un enfoque que reduzca el análisis de las prácticas al sujeto, o a las intenciones del sujeto, como de una especie de física social, que busca en las estructuras objetivas los determinantes de los individuos.

Bourdieu se presenta pues, como especialmente pertinente en un intento de superar una visión dicotómica del mobbing, en que éste intente explicarse centrándose en el sujeto (Piñuel 2001) o centrándose en la estructura (Fernández y Llorens 2002).

En el modelo psicopatológico de Piñuel (2001) el factor de más peso para explicar el mobbing es el perfil psicológico tanto del acosador/a como de la victima, y las actuaciones que se derivan de su propio perfil.

En el otro extremo, en el modelo de la Prevención de Riesgos Laborales de Fernández y Llorens (2002) el factor de más peso es la organización del trabajo, a tal punto que si el trabajo está bien organizado y no puede ser utilizado como instrumento de acoso. En palabras del propio Bourdieu:

"Se trata de escapar a la vez a la filosofía del sujeto sin sacrificar al agente, y de la filosofía de la estructura pero sin renunciar a tomar en cuenta los efectos que ella ejerce sobre el agente 0 a través de él".

(Bourdieu y Wacquant 1997: 97).

Bourdieu subraya que los agentes sociales no son ni marionetas de las estructuras, ni dueños de la misma. Desde este esfuerzo de superación y síntesis teórica, surge el proyecto de gran teoría que lleva a cabo Bourdieu de La Práctica y del Sentido Práctico. Un sentido y una lógica de la práctica que no se encuentra ni en el análisis exclusivo de la estructura ni en el del agente, sino en la relación dialéctica entre la estructura y el agente.

Planteamos que el mobbing puede explicarse también como una práctica, resultado de la interacción entre estructura y agentes. 


\section{Conceptos fundamentales de la Teoría de la Acción de Bourdieu}

\section{El concepto de "Campo"}

Podemos considerar que este concepto tiene influencias del marxismo en el sentido de que el autor plantea que el mundo está condicionado por estructuras objetivas, independientes de la conciencia y de la voluntad de los agentes, que son capaces de orientar y coaccionar sus representaciones (Bourdieu 1996: 127).

Ahora bien, ¿cómo podríamos identificar o definir un Campo? Quizá a riesgo de simplificar mucho una teoría tan compleja como la de Bourdieu, podríamos decir que un Campo está compuesto por Agentes y por Prácticas. Por lo tanto, para identificar o definir un Campo, tendríamos que identificar a los agentes que lo forman, a la red de relaciones que mantienen entre sí (Estructura del Campo) y las Prácticas específicas que en él se desarrollan. El concepto de Campo define también un espacio social y sus límites. Y pensar en términos de Campo, implica pensar relacionalmente, implica pensar el mundo social como un mundo de relaciones objetivas.

\section{La estructura del Campo}

"La realidad social' de la que habla Durkheim es un conjunto de relaciones invisibles, las mismas que constituyen un espacio de posiciones exteriores las unas a las otras, definidas unas en relación a las otras, por la proximidad, la variedad o por la distancia, y también por la posición relativa, por encima o por debajo o incluso entre".

(Bourdieu 2002a: 16).

Podemos visualizar imaginariamente un Campo como una red de relaciones objetivas entre posiciones. Al tratarse de un espacio definido por las relaciones, las posiciones se definen por oposición a otras, de forma que el valor de una posición social no se puede medir de hecho más que por "la distancia social" que la separa de otras posiciones inferiores o superiores. Las posiciones se definen de acuerdo a un tipo especial de Poder o Capital, específico de ese Campo (económico, cultural, social...), poseído por los agentes presentes en ese Campo que entran en lucha o competencia como parte de la dinámica interna del Campo (que juegan en ese Campo).

De aquí podemos subrayar dos ideas. La primera, la íntima relación existente entre Campo y Capital. El capital operativo en un Campo es el que lo define. Por ejemplo, el capital operativo en el campo económico es el dinero, pero ese no es el capital operativo o no el principal, en el campo de la creación literaria. Para construir un Campo, uno debe identificar las formas de Capital específico que operan en él y para construir las formas de capital específico, uno debe conocer el Campo. La otra idea es que al hablar del Campo como un sistema de posiciones, debemos entender que los Agentes que actúan en él -individuos, grupos o instituciones- formarán parte del Campo, siempre que posean el tipo de Capital operativo en un Campo o capital convertible en capital operativo en ese 
Campo. Y que su posición en la estructura del Campo estará relacionada con el Capital que posean. No hay posición social que puede definirse exclusivamente por sí misma. Bourdieu define más explícitamente la Estructura del Campo, al decir:

"La Estructura del Campo es un estado de la relación de fuerzas entre los agentes o las instituciones implicados en la lucha 0 , si se prefiere así, de la distribución del Capital específico que acumulado en el curso de luchas anteriores, orienta las estrategias ulteriores. Esta estructura que constituye el principio de las estrategias destinadas a transformarla, está ella misma siempre en juego: las luchas que tienen lugar en el Campo tienen por objetivo el monopolio de la violencia legítima (autoridad específica) que es característico del Campo considerado, es decir en definitiva la conservación o la subversión de la estructura en la distribución del capital específico".

(Bourdieu 2003: 113).

Al hablar de la Estructura del Campo, Bourdieu la concibe como un estado de la relación de fuerzas, por lo tanto la Estructura del Campo no es una estructura estable, sino dinámica. Los intentos por mantener la relación de fuerzas o por desestabilizarlo, es decir por mantener o trasformar la Estructura del Campo, dan lugar a luchas dentro del Campo. Quizá podríamos entender el mobbing como una táctica de quienes tienen Poder para mantener su posición eliminando a quienes creen que la amenaza (la víctima). Es decir, podríamos interpretar el mobbing como una práctica para mantener intacta la estructura del Campo. La estructura del Campo se mantiene o desequilibra dependiendo de si los agentes que ocupan las distintas posiciones, mantienen, incrementan o pierden el Capital específico del Campo que poseen, y que determina su posición. De ahí que en un principio hayamos de subrayar el aspecto dinámico y conflictual que caracteriza a todo Campo, comparable a un Campo de batalla, donde se lucha entre los diferentes agentes por monopolizar el Capital que es objeto de la lucha y por ocupar diferentes posiciones.

"Es en cada momento el estado de las relaciones de fuerza entre los jugadores lo que definen la estructura del Campo: podemos imaginar que cada jugador tiene delante montones de fichas de diferentes colores correspondientes a las diferentes especies de Capital que detenta, de forma que su fuerza relativa en el juego, su posición en el espacio de juego y sus estrategias de juego [...] dependen a la vez del volumen global de sus fichas y de la estructura de los montones de fichas, del volumen global de la estructura de su capital".

(Bourdieu y Wacquant 1997: 74-75).

\section{Funcionamiento de los Campos o el Juego de los Campos}

Aunque hemos hablado de la Estructura de los Campos, hemos de entender que un Campo es ante todo un espacio social dinámico. Y que como hemos dicho anteriormente, un Campo se define también por la Práctica concreta que se desarrolla en él. 
Bourdieu recurre a la metáfora del juego para explicar el funcionamiento de los campos.

"Para que un Campo funcione es preciso que haya objetos en juego y personas dispuestas a jugar el juego, dotadas de los Habitus que implica el conocimiento y el reconocimiento de las leyes inmanentes del juego, de los objetos en juego, etc".

(Bourdieu 2003: 113).

De este modo, un Campo está constituido por la existencia de capitales del mismo tipo y por un conjunto de estrategias de lucha que tienden a la apropiación de los mismos. En cierta manera, los campos son espacios sociales en los que unos jugadores con diferente volumen de capital específico de ese campo juegan un juego (específico de cada campo), donde las relaciones y las luchas que se establecen en el campo y las diferentes estrategias que llevan a cabo los agentes, están en función de esos capitales.

Uno de los aspectos o propiedades del Juego en un Campo que nos interesa es que la lucha de los agentes implicados queda disimulada por la confabulación entre quienes allí juegan para jugar de esa manera. Esto podría expresarse en los términos "el Juego es asi" (por ejemplo "las relaciones laborales son asi" ) naturalizando el juego y ocultando que este juego es el resultado de un acuerdo implícito entre los jugadores. Aplicado al mobbing, el proceso de des-naturalización del juego nos llevaría a que el acoso no es una consecuencia "natural" de las relaciones laborales, entendidas como relaciones de poder, sino que hay un acuerdo implícito entre determinados jugadores, que lo legítima hasta tal punto que Io "naturaliza" ( Bourdieu 2003: 114). En definitiva, varios factores son necesarios para que sea posible la existencia del juego y jugar el juego: Jugadores, Capital, Interés en el juego y el conocimiento de las Reglas del Juego que permitan jugarlo y desarrollar estrategias.

Respecto a las Reglas del Juego, es importante señalar que éstas no tienen por qué ser explícitas (Bourdieu 1996: 72). Ser conscientes de este hecho es fundamental para el estudio del mobbing, ya que nos sitúa en el terreno de las "normas no escritas", en el terreno de "lo no formal".

El concepto de "juego", sería la versión suave, por así decirlo, de lo que acontece en los campos. Como hemos dicho anteriormente, Bourdieu toma ideas centrales del marxismo, como son que la sociedad está estructurada en clases y que las relaciones sociales son relaciones de lucha. Por lo tanto, además de hablar del juego de los campos, podemos hablar de la lucha en los campos (Bourdieu 2002: 50).

\section{Campo de Poder}

Bourdieu halló que había ciertas propiedades de los Campos que no había manera de explicar con los conceptos de su teoría. Por ello, se vio obligado a revisar los mismos, y a introducir los conceptos de Campo de Poder y Campo de las Clases Sociales, para dar cuenta de determinados hechos sociales (Bourdieu 2002). En cierta manera, estos campos están presentes en todos los demás, son transversales a todos los demás. 
"El Campo de Poder (que no hay que confundir con el Campo Político) no es un Campo como los demás; es el espacio de las relaciones de fuerza entre los diferentes tipos de Capital, o con mayor precisión, entre los agentes que están suficientemente provistos de uno de los diferentes tipos de capital para estar en disposición de dominar el Campo correspondiente [...], en particular, cuando están amenazados los equilibrios establecidos en el seno del Campo de las instancias específicamente encargadas de la reproducción del Campo de Poder".

(Bourdieu 2002b: 51).

Por lo tanto, podríamos decir que la lucha en el Campo de Poder se intensifica en dos situaciones. Una, cuando los agentes dominantes consideran que sufren un ataque externo que puede alterar su posición en el Campo de Poder. Otra situación que intensifica la lucha en el Campo se da cuando los agentes dominantes consideran que sufren un ataque interno, que puede alterar su posición de poder en el Campo. Bourdieu se refiere en este segundo caso a los agentes dominantes como "aquellas instancias específicamente encargadas de la reproducción del Campo de Poder". Las identificamos con la "clase dominante" basándonos en lo que el propio Bourdieu expone:

"Los que en un estado determinado de las relaciones de fuerza monopolizan (más o menos completamente) el capital específico, fundamento del poder o de la autoridad específica característica de ese Campo, se inclinan por las estrategias de conservación".

(Bourdieu 2003: 114).

\section{El concepto de "Capital"}

Como primera aproximación al concepto, podríamos decir que el Capital es "lo que tiene valor" en el Campo, y su apropiación y control el objeto de la lucha en el Campo.

"Un Capital o una especie de Capital es aquello que es eficaz en un Campo determinado, a la vez en tanto que arma y en tanto objeto de lucha, lo que permite a su detentador ejercer un poder, una influencia, o sea, existir en un Campo determinado, en lugar de ser una simple "cantidad despreciable'".

(Bourdieu y Wacquant 1997: 74).

De esta forma, la posesión de un Capital específico de un Campo, es a la vez requisito de entrada en ese Campo, objeto de lucha y arma de lucha en ese Campo. Bordieu distingue entre cuatro tipos de capital: El Capital económico, el Capital cultural, el Capital simbólico y el Capital social. Nos interesa especialmente el Capital social, que es la capacidad para movilizar recursos vinculados a redes duraderas de relaciones, y que es convertible en capital económico o Poder. Nos interesa en el estudio del mobbing porque nos remite al tema del poder informal obtenido a través del capital social. 


\section{El concepto de "Habitus"}

El concepto de Habitus es amplio y complejo, por lo que no es posible que sea contenido, aprehendido en una sola definición, sino que es necesario realizar aproximaciones sucesivas al mismo, desde la perspectiva de sus diferentes facetas. Una introducción general al concepto, nos la ofrece el propio Bourdieu (1996):

"Las nociones que he elaborado poco a poco, como la noción de Habitus, nacieron de la voluntad de recordar que al lado de la norma expresa y explícita del cálculo racional, hay otros principios generadores de las prácticas.

Eso es sobre todo en las sociedades en las que hay pocas cosas codificadas; de suerte que para dar cuenta de lo que la gente hace, es necesario suponer que obedecen a una suerte de "sentido del juego" como se dice en deporte, y que, para comprender sus prácticas es necesario reconstruir el esquema de capitales informacionales que les permite producir pensamientos y prácticas sensatas y regladas sin intención de sentido y $\sin$ obediencia consciente a reglas explícitamente enunciadas como tales".

(Bourdieu 1996: 83).

\section{El Habitus como sistema de disposiciones}

Bourdieu, también define el Habitus como sistema de disposiciones. De manera más concreta:

"El Habitus, sistema de disposiciones adquiridas por aprendizaje implícito o explícito que funciona como un sistema de esquemas (schèmes) generativos [...] Se lo puede pensar por analogía con un programa de ordenador (analogía peligrosa por mecanicista), pero un programa capaz de autocorregirse. Está constituido por un conjunto sistemático de principios simples y parcialmente sustituibles, a partir de los cuales se puede inventar una infinidad de soluciones".

(Bourdieu 2003: 119).

El Habitus es a la vez producto de la práctica y principio o matriz generadora de prácticas, de las percepciones, apreciaciones y acciones de los agentes. El Habitus supone la posibilidad de construir una determinada Teoría de la Práctica, como producto de un "sentido práctico" adquirido por la práctica del juego. En palabras del propio autor:

"El Habitus es esa especie de sentido práctico de lo que hay que hacer en una situación determinada".

(Bourdieu 2002b: 40).

El concepto de Habitus nos plantea la cuestión de la consciencia sobre los actos realizados. Bourdieu (2002: 40) defiende que no debemos confundir el Habitus con lo que 
otros llamarían inconsciente.

"Los 'sujetos' son en realidad agentes actuantes y conscientes dotados de un sentido práctico, sistema adquirido de preferencias, de principios de visión y de división (lo que se suele llamar un gusto) de estructuras cognitivas duraderas que esencialmente son fruto de la incorporación de estructuras objetivas y de esquemas de acción que orientan la percepción de la situación y la respuesta adaptada". (cursiva nuestra)

(Bourdieu 2002b: 40).

Podríamos decir que el Habitus supone una tendencia o manera de actuación dentro de la cual existen elecciones. Bourdieu defiende que con su noción de Habitus intenta eludir el mecanicismo propio del inconsciente y los vacíos que este deja en el análisis social. Es decir, parece que la acción de los agentes sólo puede explicarse desde una dicotomía entre la elección racional y el inconsciente individual. Como alternativa, el autor propone el concepto de Habitus.

"Las condiciones del cálculo racional no están dadas prácticamente nunca en la práctica: el tiempo es contado, la información limitada, etc. $Y$ sin embargo, los agentes hacen, mucho más a menudo que si procedieran al azar, 'lo único que se puede hacer'. Esto porque abandonándose a las intuiciones del "sentido práctico" que es el producto de la exposición durable a las condiciones semejantes o a aquéllas en las cuales están colocados, anticipan la necesidad inmanente al curso del tiempo".

(Bourdieu 1996: 24).

El Habitus es matriz de la práctica, pero no se puede hacer de ello el principio exclusivo o automatizado de toda práctica. De alguna manera, Bourdieu define también su concepto de "agencia", al definir el concepto de Habitus. El Habitus da respuesta a la pregunta ¿qué hay que hacer ahora?, más que a preguntas del tipo ¿por qué hago esto?

Es decir, en muchos casos no sé cuál es la razón última o profunda. Pero esto se hace así porque funciona. Es decir, la razón es que "es práctico".

\section{El Habitus y el Campo}

El concepto de Habitus está estrechamente ligado con el de Campo, ya que existe una relación intensa entre las posiciones sociales (Estructura del Campo), las disposiciones (Habitus) y las tomas de posición (las prácticas).

"El Habitus es a la vez un sistema de esquemas de producción de prácticas y un sistema de esquemas de percepción y de apreciación de las prácticas. Y en los dos casos, sus operaciones expresan la posición social en la cual se han construido".

(Bourdieu,1996: 134). 
En El sentido práctico (Bourdieu 1991) el autor ya definía los Habitus en relación a los campos. El Habitus es una estructura estructurada, producto del Campo que lo genera. Pero a su vez el Habitus es una estructura estructurante, ya que es la matriz generadora que organiza las percepciones y actuaciones de los agentes y determinan y orientan sus prácticas.

El Habitus como estructura estructurada consiste en un conjunto de disposiciones a percibir, pensar, sentir y actuar de determinada manera, adquiridas mediante el proceso de socialización. De esta forma, el Habitus también puede ser entendido como resultado de la interiorización de una cultura o subcultura. El Habitus depende de la posición que los agentes ocupan en el Campo, de las dinámicas de funcionamiento del Campo (del juego del Campo) y de la acción pedagógica que el Campo ejerce sobre sus agentes (proceso de socialización intenso o no). El Habitus es, pues, como estructura estructurada, un producto social.

El Habitus como estructura estructurante es una producción social, una especie de "piloto automático" que nos permite "saber lo que hay que hacer", aunque no sepamos muy bien por qué en cada momento. Recordamos que el Habitus es una especie de gramática generativa, que puede articular diferentes respuestas según el contexto. Como hemos dicho anteriormente, sería como si habiendo interiorizado la gramática musical, pudiéramos exteriorizar (producir) diferentes melodías de acuerdo con el contexto (por ejemplo diferentes formas de ejercer el Poder).

Aunque marginalmente, casi como un comentario casual, Bourdieu plantea una cuestión que nos interesa: ¿qué pasa cuando un agente no puede desarrollar su Habitus porque la situación del Campo no lo permite? ¿Por ejemplo su Habitus de ejercicio del Poder?

'La 'situación' es en cierta manera, la condición de posibilidad de una realización conseguida del Habitus.

Cuando no se dan las condiciones objetivas para una realización lograda, el Habitus contrariado y continuamente, por la situación puede alojar fuerzas explosivas (resentimiento), que pueden esperar (incluso acechar) la ocasión para actuar y que se expresan a partir del momento en que las condiciones objetivas (posición de poder del pequeño jefe) le ofrecen la posibilidad de hacerlo... (El mundo es una enorme reserva de violencia acumulada, que se revela cuando encuentra las condiciones para su realización)".

(Bourdieu 2003: 134).

Por lo tanto, podríamos entender el mobbing, como parte de un Habitus. El Habitus del ejercicio del Poder, al verse contrariado para cristalizar en su forma habitual, espera "la situación" para cristalizar en una forma nueva, violenta, a través del mobbing. En todo caso, Bourdieu, plantea una cuestión que nos interesa: las modificaciones en "la situación" (en el Campo), influyen en el desarrollo del Habitus.

\section{Habitus colectivo}

Aunque el Habitus se expresa de manera individual, es en realidad algo colectivo. Hablar de Habitus es hablar de lo individual y personal como colectivo y social. El Habitus es la 
socialización de la historia colectiva. "Producto de la historia, el Habitus produce prácticas, individuales y colectivas" (Bourdieu 1991: 91).

Los Habitus individuales son la concreción individual de Habitus colectivos cuando comparten características similares. La similitud de estos Habitus individuales es producto de la similitud de las condiciones de existencia en que se fraguan, de la situación en un momento histórico del Campo y de la posición que los agentes ocupan en él. Bourdieu (2002: 19) explica que una de las funciones del concepto de Habitus es que permite dar cuenta de la unidad de estilo que une las prácticas y los bienes de un agente singular o de un grupo de agentes. El concepto de Habitus colectivo posee un gran potencial para analizar la cultura de acoso de la empresa.

\section{Habitus y cambio}

Como ya hemos visto, el Habitus es un sistema de disposiciones duraderas. También hemos visto que las prácticas se producen en el encuentro entre el Habitus y el Campo. El Habitus funciona con relación al Campo social y produce o genera prácticas diferentes según lo que acontece en el Campo. Por eso mismo es necesario plantearse en qué tipo de Campo el Habitus es eficaz.

Aplicado a nuestro caso concreto, si el Habitus del acoso no es eficaz en el Campo porque el Campo castiga ese Habitus (sanciones, proceso de criminalización...) podemos deducir que hay posibilidades de que esta práctica - si no desaparece- se reduzca porque deja de ser práctica. En términos del autor: "La lógica de la práctica es ser lógica hasta el punto donde ser lógico cesaría de ser práctico" (Bourdieu 1996: 86).

Luego para modificar un Habitus quizá sea necesario intervenir en el Campo modificando el juego del campo. Mientras un Habitus sea funcional, es decir, práctico y operativo en un Campo, se mantendrá, y cuanto más operativo y práctico sea, más resistente será al cambio.

\section{El concepto de "la Práctica"}

Recordamos que en su Filosofía o Teoría de la Práctica, Bourdieu intenta realizar una superación de aquellas posturas que defienden el primado de la estructura sobre el agente y al revés, el primado del agente sobre la estructura.

Bourdieu plantea que la Práctica y el sentido práctico surgen de la relación dialéctica entre la estructura y el agente. Nuestra propuesta concreta es que podemos analizar el mobbing como una Práctica resultado de la interacción de una serie de factores que podemos encuadrar dentro de los conceptos de Habitus, Capital y Campo. El modelo de Bourdieu puede actuar como un modelo de mayor potencia teórica, que incluya factores explicativos de los modelos explicativos específicos del mobbing, así como factores no incluidos porque estos no incluyen. 


\section{Metodología y Técnicas}

\section{Metodología}

Para contrastar empíricamente nuestra propuesta, es decir, la utilidad y parcialidad de los modelos de Piñuel (2001), Corsi y Peyrú (2002) y Fernández y Llorens (2003), a la vez que la mayor potencia explicativa de nuestro modelo teórico, procedemos a aplicar todos los modelos al análisis de un mismo caso, y a comparar los resultados respecto a la información y comprensión sobre el caso que aporta cada uno de ellos.

Xavier Coller (2000) nos recuerda que la generación de conocimiento en Sociología, basada en el estudio de un solo caso, tiene importantes precedentes. Así Durkheim en Las formas elementales de la vida religiosa (1912) decide analizar la tribu de los Arunta para explicar el sentimiento religioso, de forma que los Arunta se convierten en un caso estratégico y único. Asimismo, Weber publica en 1904 La ética protestante y el espíritu del capitalismo con el subtítulo de "Un estudio del caso de Calvino, su secta de Ginebra y la difusión de sus ideas sobre la salvación". Para demostrar sus hipótesis, analiza el caso de Richard Baxter, al que considera un tipo ideal, aunque real.

En el estudio de casos la elección del caso es una decisión metodológica fundamental, por eso ha de realizarse siguiendo los criterios de "representatividad analítica" y "pertinencia teórica".

Respecto a la "representatividad analítica", ésta hace referencia a que si hemos elegido adecuadamente un caso, los resultados de su estudio no se pueden extrapolar a un universo (representatividad estadística) sino al conjunto de teorías a las que el caso se dirige, con las que el caso está relacionado. En este sentido, señala Coller (2000):

"Si un caso está bien arropado teóricamente, se puede hacer una generalización analítica. Por ejemplo, se puede estudiar un caso que contradiga un cuerpo teórico consolidado y contribuir así a enmendar una teoría en cuestión".

(Coller 2000: 77).

Consideramos que al aplicar el modelo de Bourdieu, que permite incorporar al análisis del mobbing factores que los demás modelos no incorporan, como el Poder (Capital), la Cultura de la Empresa (Habitus) o el Contexto (Campo), mostramos que se genera una mayor comprensión del caso del mobbing que aplicando para su análisis los otros modelos explicativos (Piñuel 2001; Corsi y Peyrú 2003; Fernández y Llorens 2002). Consideramos que esta conclusión sí es generalizable.

Es decir, dado que está fuera de duda la potencia explicativa del modelo de Bourdieu para explicar otras realidades sociales, nuestro planteamiento es que si éste modelo tiene mayor potencia explicativa para el caso de mobbing que los otros modelos, también la tiene para explicar otros casos de mobbing. Estableciendo un paralelismo con las ciencias naturales, y por poner un ejemplo, no estamos estudiando células (casos) 
sino microscopios (modelos teóricos). Por lo cual, si aplicando varios microscopios para ver la misma célula, hay uno que nos permite verla mejor, podemos decir que con ese microscopio se ve mejor que con los demás y, por lo tanto, recomendar su uso para el estudio de esas células.

El que el caso sea adecuado depende de su "pertinencia teórica". Un caso resulta adecuado cuando nos permite dialogar con las teorías a las que se dirige, para ensancharlas, refutarlas, modificarlas o comprobarlas. Coller (2000) pone el ejemplo de la relación entre modernización y fundamentalismo islámico, para el estudio del cual sería más adecuado el estudio del caso de Argelia que el caso de Chile.

El caso elegido es especialmente pertinente teóricamente porque pasa por tres fases, en cada una de los cuales el Poder de los acosadores varía y varía el mobbing: en la primera, los acosadores tienen Poder (formal e informal) y ejercen el mobbing sobre la víctima; en la segunda, los acosadores pierden el Poder formal y parte del informal y disminuye y casi desaparece el mobbing; y en la tercera, los acosadores recuperan el Poder formal y el informal y se reinicia el mobbing. El caso era muy pertinente para demostrar la centralidad del Poder en el mobbing, y la necesidad de incorporarlo de forma fuerte al análisis del fenómeno incluyéndolo dentro de cualquier modelo explicativo.

Asimismo, se trata de un caso con una duración de ocho años, que se ha estudiado durante cinco años, y que ha estado varias veces en la vía judicial, lo que ha generado abundante documentación. La copiosa información, permitía aplicar todos los modelos de análisis del mobbing al caso (Piñuel 2001; Corsi y Peyrú 2003; Fernández y Llorens 2002), así como el modelo propuesto por Bourdieu (2002), además de permitir estudiar la influencia del Poder, el contexto y la cultura de la empresa en el surgimiento y mantenimiento del mobbing.

\section{Técnicas}

Se ha elegido un caso de una empresa pública de ámbito estatal, con una duración de 8 años, que se ha seguido y estudiado a lo largo de 5 años.

Como técnicas se han usado, en primer lugar, la recogida y estudio de documentación, con un total de 58 documentos, muchos de ellos oficiales, entre los que se incluyen: un informe detallado de Inspección de Trabajo sobre el caso de mobbing (28 páginas), sentencias judiciales, evaluaciones de riesgos laborales, denuncias de varios sindicalistas a Inspección de Trabajo, respuestas de Inspección de Trabajo, escritos de sindicalistas y de la víctima a la dirección de la empresa (con firma y sello de registro de entrada de la empresa), escritos de la Dirección a la víctima (con firma manuscrita y sello de registro de salida de la empresa), denuncia de la víctima por amenaza de muerte a la Guardia Civil (con número de registro de entrada y sello de la Comandancia), escritos al Defensor del Pueblo de España, respuesta del Defensor del Pueblo, así como variada documentación interna de la empresa relacionada con el caso (reparto de la plantilla, horarios, órdenes de trabajo, escritos de los acosadores contra la víctima firmados y puestos en los tablones de la empresa). 
En segundo lugar se realizaron entrevistas abiertas en profundidad en la fase exploratoria a los principales implicados en el caso (8 entrevistas) y entrevistas semiestructuradas en la fase de recogida de información (8 entrevistas) con un total de 16 entrevistas. Entre las limitaciones de la investigación está la de no poder entrevistar a los acosadores ni a sus jefaturas, algo habitual en las investigaciones de mobbing. La falta de información de estos actores queda suplida con la información proporcionada sobre el caso por Inspección de Trabajo y por la posibilidad de contrastar las informaciones obtenidas de las entrevistas con otros actores, con la abundante información documental generada alrededor del caso.

Finalmente se recogió material gráfico relacionado con el caso, como fotografías, grabaciones televisivas y prensa local.

La abundante información y los diferentes tipos de la misma posibilitó una buena triangulación.

\section{Principales resultados}

Presentamos a continuación el modelo propuesto y las potencialidades para el análisis que presenta frente a los modelos preexistentes. Con el fin de que pueda verse más claramente la capacidad de los conceptos de Bourdieu para incorporar factores cuya importancia en el mobbing ya habían sido detectados por Piñuel (2001), Corsi y Peyrú (2003) y Fernández y Llorens (2002), así como para incorporar factores nuevos, en algunos momentos de la explicación pondremos ejemplos de la investigación empírica realizada.

El concepto de "Campo" nos permite incorporar el contexto al análisis del mobbing. Simplificando mucho, Bourdieu plantea que el mundo está condicionado por estructuras objetivas, independientes de la conciencia y de la voluntad de los agentes, que condicionan sus representaciones (Bourdieu 1996: 207), pero que a su vez, los agentes no son ni marionetas de las estructuras, ni dueños de la misma (Bourdieu y Wacquant 1997: 97).

El Campo consta de tres dimensiones: Agentes, Estructura del Campo y Prácticas del Campo, que nos permiten operacionalizar el análisis del contexto.

La dimensión de los Agentes nos permite incorporar las aportaciones de Piñuel (2001) al estudio del mobbing, quien considera que el factor fundamental en el mobbing son los perfiles personales del acosador y de la víctima. Pero a su vez, nos permite superar esta visión del mobbing como un asunto acosador/a -víctima, e incorporar las aportaciones de otros autores sobre los actores implicados, como la cadena de mando (NTP 476, Martín Daza y otros 1998), las secciones sindicales y los sindicatos (Fernández y Llorens 2002) o actores no previstos como Inspección de Trabajo, o en los casos de empresas o administraciones públicas, agentes de otras instituciones públicas distintas a aquéllas en las que se da el caso de mobbing.

La dimensión de la Estructura del Campo, hace referencia a las posiciones que ocupan los agentes en una red de relaciones (Bourdieu 2002: 16) teniendo en cuenta que esta red no es estática sino dinámica, ya que su estructura es el resultado de la relación de fuerzas y de las luchas que mantienen los diferentes agentes que forman parte de ella (Bourdieu 2000: 113; Bourdieu y Wacquant 1997: 74-75). 
Gráfico 2.

Modelo de análisis propuesto.

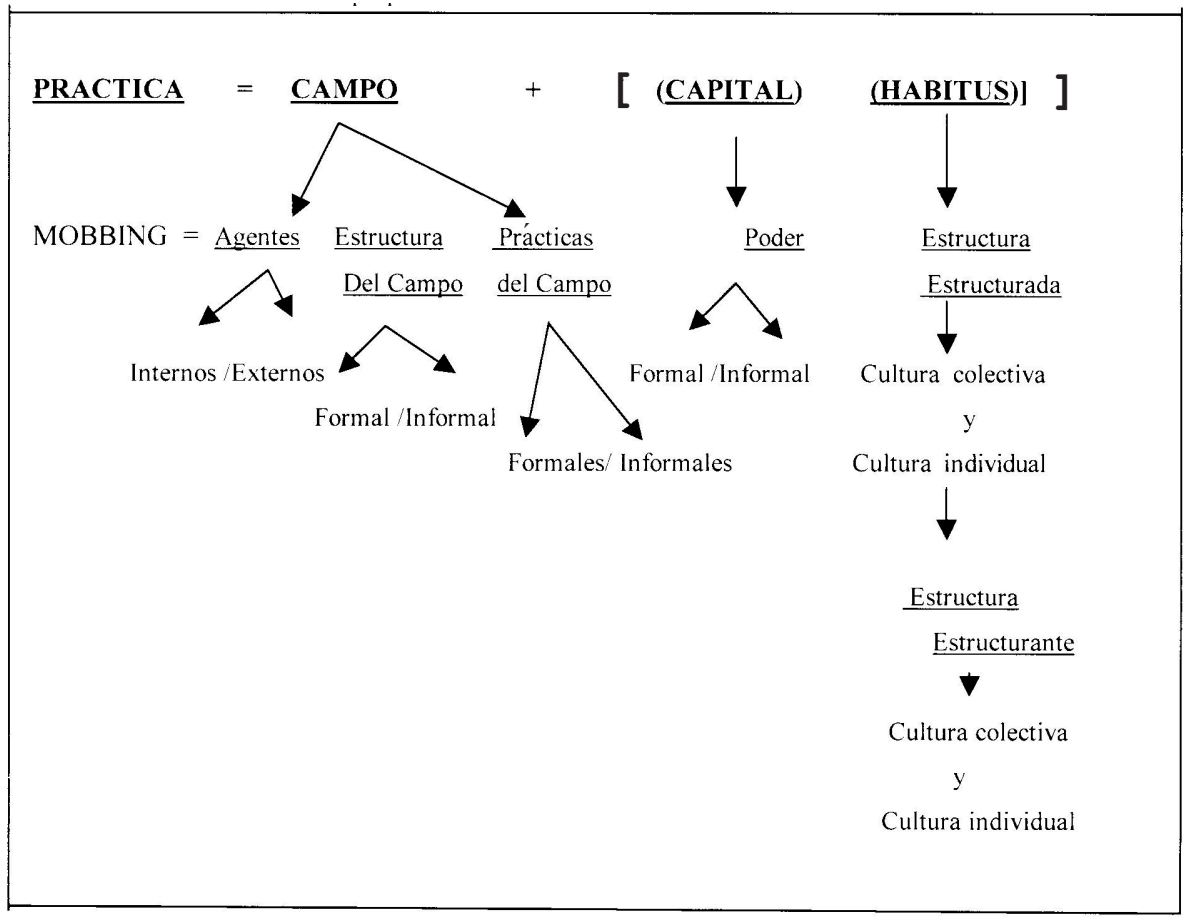

La Estructura del Campo nos permite una primera integración del factor Poder al análisis del mobbing, uno de nuestros objetivos. La posición de Poder, formal e informal, que ocupan tanto el acosador/es como la víctima dentro de una red de relaciones, aparece como un factor de peso en el desarrollo del mobbing.

Otra potencialidad importante de la dimensión Estructura del Campo, es que nos permite entender el mobbing como un medio (una Práctica) desarrollada por aquellos agentes que tienen poder -formal e informal- para mantener su posición, eliminando a quienes consideran que la ataca (la víctima del mobbing). Es decir, dado que según Bourdieu la Estructura del Campo es inestable, porque es producto de la relación de fuerzas entre los Agentes presentes en el Campo, podemos entender que el mobbing es un medio usado por los que Bourdieu (2003: 114) llama dominantes (los que tienen el poder en un Campo) para mantener su posición de Poder en el Campo y subsidiariamente, la estructura del Campo.

Esta lectura del mobbing, también nos permite explicar la implicación de la cadena de mando apoyando o encubriendo a los acosadores (NTP 476; Martín Daza y otros 1998). Puesto que forman parte de los dominantes, al apoyar o encubrir al acosador/a, 
no hacen más que defender la Estructura del Campo existente, y su propia posición de Poder en la misma. Para entender esto, hemos de recordar que ninguna posición en el Campo, se define por sí misma, sino que se definen unas en relación a las otras (Bourdieu 2002: 16), por lo que una alteración en una posición en el Campo (por ejemplo que el acosador pierda el poder formal) afecta a todo el Campo (por ejemplo a todos los agentes del campo que construyen su poder informal construyendo alianzas con el acosador). La dimensión de Estructura del Campo también nos permite explicar la implicación de otros trabajadores (del mismo nivel o inferior) en el acoso. Al contribuir al acoso están colaborando en el mantenimiento de una Estructura del Campo que les beneficia. Por ejemplo en el caso estudiado, aquellos trabajadores que participaban en el acoso obtenían de los acosadores (Jefaturas) una serie de beneficios materiales (horas extras que cobraban, buenos horarios, viajes con dietas) y simbólicos (algunos eran ascendidos a puestos de designación directa), en detrimento de quienes apoyaban a la víctima (se les castigaba utilizando la organización del trabajo).

Esta interpretación del mobbing como práctica para defender la Estructura del Campo y su posición por parte de los dominantes, también nos permite entender por qué aunque existan medidas explícitadas y formales para intervenir en los casos de mobbing en la empresa (protocolos específicos de intervención, artículos del Convenio Colectivo) estos no se aplican. Porque el aplicarlos (por ejemplo sancionar a un Jefe y dar la razón a un trabajador), supone alterar las relaciones de Poder entre dominantes y dominados en la empresa y por lo tanto alterar la estructura del Campo.

La dimensión de las Prácticas del Campo nos permite integrar aquellas aportaciones sobre las prácticas formales e informales en relación a la organización del trabajo y a las relaciones laborales, que pueden actuar como caldo de cultivo del mobbing (NTP 476 1998; Piñuel 2001; Fernández y Llorens 2002). Pero además de las prácticas descritas por estos autores, nos permite integrar todas aquéllas no descritas y específicas del caso analizado. Por ejemplo, en el caso estudiado había un gran número de prácticas informales respecto a las relaciones laborales que influían en la aparición y desarrollo de los casos de mobbing en la empresa.

El concepto de Capital, hace referencia a "lo que tiene valor en un Campo", es aquello que permite a quien lo posee ejercer un poder, una influencia o formar parte de un Campo determinado; es un arma para luchar en el Campo, a la vez que el conseguir poseerlo, es lo que desencadena las luchas en el Campo (Bourdieu y Wacquant 1997: 74).

El concepto de Capital nos permite integrar la aportación de Fernández y Llorens (2002) respecto a la importancia de los riesgos psicosociales como instrumento de acoso, ya que efectivamente la capacidad de los acosadores de exponer a la víctima de forma frecuente, focalizada y prolongada a los riesgos psicosociales, se convierte en un Capital para ejercer el acoso.

Pero la gran potencialidad del concepto de Capital es que nos permite integrar plenamente el factor Poder al análisis del mobbing, uno de nuestros objetivos, ya que considerábamos que era una deficiencia importante en todos los modelos revisados.

En la investigación realizada como parte de la Tesis (Mayoral Blasco 2007) pudimos 
comprobar la centralidad del Poder en la aparición y el mantenimiento del mobbing, hasta el punto de que una disminución en el poder - formal e informal— de los acosadores, supone una disminución o incluso el cese del acoso. Esto señala una línea de intervención en los casos, ya que muchos casos se "resuelven" cambiando a la víctima de puesto de trabajo y mandando a otro trabajador/a con el acosador, con lo que en muchos casos se vuelve a repetir el acoso, tal como señala Piñuel (2001)'. En estos casos no se cambian las condiciones estructurales para que el acoso no se vuelva a repetir, que tampoco pasarían por cambiar de puesto al acosador, sino por dejarlo sin Capital para ejercerlo (sin Poder). Evidentemente, se pueden tomar con mayor facilidad medidas destinadas a la disminución del poder formal (como degradación funcional, despido), que a la disminución del poder informal, ya que el poder informal de los agentes es de difícil control. Para controlar el poder informal de los acosadores, quizá sería necesario tomar otras medidas, como un proceso de criminalización de los acosadores/as y de estas prácticas.

El concepto de "Habitus" es amplio y complejo. En una de las varias definiciones que proporciona de este concepto, Bourdieu (2002: 40), lo define como un sistema de disposiciones adquiridas por aprendizaje implícito o explícito, que funciona como un sistema de esquemas generativos; lo compara (advirtiendo de que la comparación es peligrosa por mecanicista) con un programa de ordenador, pero capaz de autocorregirse). Bourdieu (1996: 134) también explica su concepto de Habitus diciendo que de alguna manera sería como si habiendo interiorizado una gramática musical (el Habitus como estructura estructurada) pudiéramos producir diferentes melodías de acuerdo con el contexto (el Habitus como estructura estructurante).

El concepto de Habitus, es también una de las grandes aportaciones del modelo al análisis del mobbing. Este concepto nos permite integrar la cultura de la empresa al análisis, factor clave según varios autores (Agencia Europea para la Seguridad y la Salud en el Trabajo 2002; Pérez Bilbao 1998; 2001; 2002; Sáenz Navarro 2001). Para operacionalizar el análisis de la cultura, recurrimos a las aportaciones de Denisse Rosseau (1998) quien señala que todos los autores coinciden en que los componentes de la cultura son cinco: presunciones básicas, valores, normas de comportamiento, patrones de comportamiento, y símbolos.

El concepto de Habitus en su dimensión de estructura estructurada, nos permite detectar como la cultura de acoso individual y colectiva, existente en la empresa influye en la aparición del mobbing. La dimensión del Habitus como estructura estructurante nos permite detectar por qué mecanismos esta cultura de acoso (y el propio acoso) se reproducen en la empresa.

\footnotetext{
1 Estudiamos un caso en la tesina (Mayoral Blasco, 2003), en que mediante este procedimiento, el acosador había acosado a cinco personas seguidas, todas ellas con baja médica con síndrome de ansiedad y depresión. Aunque toda la empresa lo sabía, especialmente el Departamento de Personal, no se intervino en ninguno de los casos.
} 
En la investigación realizada, para analizar el Habitus en su dimensión de estructura estructurada, es decir la cultura existente en la empresa que puede fomentar la aparición del acoso, ha sido necesario recurrir a las aportaciones de otros autores como Gareth Morgan (1990) y Henry Mintzberg (1998) ya que pudimos detectar que en la empresa se jugaban un gran número de juegos de poder (como el juego de Construcción de Imperios que tenía una gran importancia en el caso). Las aportaciones de estos autores nos permitieron ver que el mobbing era un juego de poder más (un juego de Mando) de los que se jugaban en la empresa, y que no podíamos entender el mobbing descontextualizado de ellos.

Es decir, el concepto de Habitus en su dimensión de estructura estructurada es lo suficientemente potente como para integrar las aportaciones teóricas de otros autores, necesarias para poder analizar el caso con profundidad.

Para analizar el Habitus en su dimensión de estructura estructurante, es decir, en nuestro caso cómo se reproduce la existencia del mobbing en la empresa, resultan muy útiles las aportaciones de Corsi y Peyrú (2003). Estos autores describen mecanismos como el encubrimiento de la violencia (citado por la NTP 476, Martín Daza y otros 1998; Piñuel 2001), pero además otros muchos como la negación de la violencia o la insensibilización. Es decir, esta subdimensión es lo suficientemente amplia para permitirnos integrar las aportaciones de otros teóricos, necesarias para analizar en un caso como se reproduce la cultura de acoso, individual y colectiva, en la empresa.

El concepto de Habitus también ha sido fundamental para entender la verdadera naturaleza del mobbing. Mucha de la literatura revisada considera que las organizaciones laborales han de funcionar como un todo armónico con un único objetivo común de producir eficientemente, por lo cual el mobbing es una "disfunción" del sistema. Frente a ella la perspectiva política del análisis de las organizaciones laborales dentro de la cual nos situamos (Manuel Alcaide Castro 1987; Morgan 1990; Mintzberg 1998) nos proporciona una visión de las organizaciones laborales como la de un conjunto de actores, con diferentes recursos (entre ellos el Poder), que persiguen sus propios objetivos, lo cual genera conflicto.

Bourdieu (2002: 40; 1996: 24; 1996: 86) defiende que una de las características de un Habitus es la de ser práctico, y que un Habitus se produce y se reproduce mientras resulta ser práctico. Desde esta perspectiva hemos podido ver que el mobbing, no es una disfunción del sistema, sino que al contrario, es una función del sistema. El mobbing se produce y se reproduce en la organización laboral porque es funcional (en términos de Bourdieu, es Práctico) para quienes lo ejercen, ya que sin apenas costes (no se sanciona), permite obtener importantes beneficios (como mantener la posición de poder y los beneficios materiales y simbólicos que de ella se derivan). En el caso estudiado, el mobbing era práctico para los acosadores, para los superiores jerárquicos y para los trabajadores que participaban en el mismo. Todos obtenían algún beneficio al acosar a la víctima, y sin ningún coste, hasta que empezó la intervención del sindicato en el caso. Ello abre las puertas a la intervención, ya que una manera de intervenir sobre el mobbing, es llevar a acabo actuaciones que hagan que éste deje de ser funcional, es decir 
que tenga costes muy importantes para quienes lo realizan, lo que influiría tanto en su práctica individual como en su práctica colectiva, como parte de la cultura de la empresa.

\section{CONCLUSIONES}

Los conceptos del modelo de Bourdieu de Campo, Capital y Habitus son lo suficientemente potentes como para que se puedan incorporar los factores que otros autores habían detectado que estaban relacionados con el mobbing e incluido en sus modelos, (Piñuel 2001; Corsi y Peyrú 2003; Fernández y Llorens 2002). Pero los conceptos de Bourdieu también nos han permitido incorporar de forma fuerte y dentro del modelo de análisis, el Poder, una factor considerado clave por todos los autores de referencia en el tema (y que estos modelos no incorporaban de forma satisfactoria). A su vez, estos conceptos nos han permitido incorporar al análisis del mobbing factores también considerados importantes como son el contexto y la cultura del empresa.

El modelo de Bourdieu da cuenta del mobbing en mayor medida que lo que lo hacen los modelos citados, con lo que alcanzamos así uno de nuestros objetivos iniciales. proporcionar un modelo teórico con una mayor capacidad explicativa del mobbing.

\section{REFERENCIAS BiBLIOGRÁFICAS}

Agencia Europea para la Seguridad y la Salud en el Trabajo. 2002. "Acoso moral en el Trabajo." Hoja Informativa FACTS 23, Bruselas.

Alcaide Castro, M. 1987. Conflicto y Poder en las organizaciones. Madrid: Ministerio de Trabajo y Seguridad Social.

Bourdieu, P. 1990. Sociología y Cultura. México: Consejo Nacional para la Cultura y las Artes.

Bourdieu, P. 1991. El sentido práctico. Madrid: Taurus.

Bourdieu, P. 1996. Cosas Dichas. Barcelona: Gedisa.

Bourdieu, P. 1998. La Distinción. Madrid: Taurus.

Bourdieu, P. 2002a. Lección sobre la Lección. Barcelona: Anagrama.

Bourdieu, P. 2002b. Razones Prácticas. Sobre la Teoría de la Acción. Barcelona: Anagrama.

Bourdieu, P. 2003. Cuestiones de Sociología. Madrid: Istmo.

Bourdieu, Pierre y Löic Wacquant.1994. Por una sociología reflexiva. Barcelona: Herder. 
Colter, Xavier (2002). Estudio de casos. Cuadernos metodológicos del CIS, n 30. Madrid: CIS.

Corsi, Jorge y Graciela María Peyrú. 2003. Violencias Sociales. Barcelona: Ariel.

Fernández Dolores y Clara Llorens. 2002. "Acoso Moral (mobbing): Una dimensión del Riesgo psicosocial." Consultado 18 de diciembre de 2002 (http./www.ccoo.es/istas).

Martín Daza, F., Jesús Pérez Bilbao y J. A. López García-Silva. 1998. "Nota Técnica de Prevención 476." El hostigamiento psicológico en el trabajo: Mobbing. Instituto Nacional de Seguridad e Higiene en el Trabajo (INSHT): Ministerio de Trabajo y Asuntos Sociales.

Mayoral Blasco, S. 2003. "Mobbing y Acción Sindical". Tesina. Facultad de Ciencias Políticas y Sociología, Universidad Autónoma de Barcelona. (Inédita. En depósito en la Biblioteca de Ciencias Sociales de la UAB).

Mayoral Blasco, S. 2007. "Mobbing, modelos explicativos y acción sindical." Tesis Doctoral. Departamento de Sociología, Universidad Autónoma de Barcelona. (Inédita. En depósito en la Biblioteca de Ciencias Sociales de la UAB).

Mintzberg, H. 1992. La estructura de las organizaciones. Barcelona: Ariel.

Morgan, G. 1990. Imágenes de la organización. Madrid: Ra-ma.

Pérez Bilbao, J. 2002. "Algunos apuntes sobre Mobbing." Consultado 6 de diciembre de 2002 (http//I www.mtas es / inshtml).

Pérez Bilbao, J., C. Nogareda Cuixart, F. Martín Daza y T. Sancho Figueroa. 2001. Mobbing, violencia física y Acoso Sexual. Madrid. Instituto Nacional de Seguridad e Higiene en el Trabajo: Ministerio de Trabajo y Asuntos Sociales.

Piñuel y Zabala, I. 2001. Mobbing: cómo sobrevivir al acoso psicológico en el trabajo. Santander: Sal Terrae.

Rosseau, D. 1998. "Clima y Cultura de Trabajo." Pp. 41-42 en Enciclopedia de Salud y Seguridad en el Trabajo, vol. II. Organización Internacional del Trabajo (OIT). Madrid: Ministerio de Trabajo y Asuntos Sociales.

Saénz Navarro, M. ${ }^{\text {a }}$ C. 2001. "Algunas cuestiones sobre el mobbing en el trabajo. Comentario a la STSJ de Navarra del 30 de abril del 2001." Aranzadi Social 10: 4551.

SUSANA MAYORAL BLASCO es licenciada en Sociología y Doctora en Sociología por la Universidad Autónoma de Barcelona en 2007, con la Tesis "Mobbing, Modelos explicativos y Acción Sindical". Es profesora asociada en la Universidad de Zaragoza, donde imparte Sociología en la Diplomatura de Relaciones Laborales y miembro del grupo de investigación consolidado "Riesgo e Integración Social" del Departamento de Sociología de la Universidad de Zaragoza.

RECIBIDO: 20/05/08

ACEPTADO: $17 / 04 / 09$

Publicado on-line: 26 de abril de 2010 